\section{Óbitos por desnutrição em idosos, São Paulo e Rio de Janeiro. Análise de séries temporais. 1980-1996}

\author{
Deaths due to malnutrition in the \\ elderly, São Paulo e Rio de Janeiro. \\ Time series analyse. 1980-1996.
}

\section{Ubirani Barros Otero}

Departamento de Epidemiologia e Métodos Quantitativos em Saúde Escola Nacional de Saúde Pública/ Fundação Oswaldo Cruz - RJ Endereço para correspondência/Correspondence to:

Rua Leopoldo Bulhões, 1480

21041-210 - Manguinhos - Rio de Janeiro

ubirani@infolink.com.br

\section{Suely Rozenfeld}

Departamento de Epidemiologia e Métodos Quantitativos em Saúde Escola Nacional de Saúde Pública/ Fundação Oswaldo Cruz - RJ

\section{Angela Jourdan Gadelha}

Departamento de Epidemiologia e Métodos Quantitativos em Saúde Escola Nacional de Saúde Pública/ Fundação Oswaldo Cruz - RJ

\section{Resumo}

Objetivos: Descrever a evolução da mortalidade por desnutrição em idosos nas Regiões Metropolitanas dos Estados do Rio de Janeiro (RMRJ) e São Paulo (RMSP), verificar as suas tendências, entre 1980 e 1996, e propor um modelo que permita prever a ocorrência de casos.

Metodologia: Trata-se de um estudo ecológico, de séries temporais, baseado em dados secundários. Foram incluídos no estudo indivíduos com 60 anos de idade ou mais, de ambos os sexos, das RMRJ e RMSP. A fonte de dados utilizada foi o Sistema de Informações sobre Mortalidade (SIM). Analisou-se a série de casos mensais, no período de janeiro de 1980 a dezembro de 1996. Para modelagem da série e predição de casos por desnutrição em idosos, utilizou-se o método Box and Jenkins, SARIMA (Sazonal Autoregressive Integrated Moving Average).

Resultados: Os resultados apontam a existência de sazonalidade, com maior número de óbitos nos meses de junho e julho na RMSP, no mês de janeiro na RMRJ, provavelmente, decorrente do clima frio, nestes meses, em São Paulo e do calor intenso no Rio de Janeiro. Este resultado pode ser explicado pela possibilidade de idosos, com estado nutricional comprometido, terem dificuldades em manter a temperatura corporal normal durante os meses de inverno, representando um estresse adicional a um organismo sem reservas.

Conclusões: As séries apresentaram tendência de aumento e revelaram um padrão sazonal no inverno na RMSP e no verão na RMRJ. Ao estimar os modelos SARIMA para as Regiões para o ano de 1996, os resultados reforçaram a hipótese de que este método pode ser eficiente para fazer previsões futuras. Provavelmente um estudo que leve em consideração causas múltiplas de morte permitirá verificar a real contribuição da desnutrição como causa básica ou contributiva para a morte. Infelizmente, até este momento, esses dados não estão disponíveis para análise.

Palavras-chave: Desnutrição. Idoso. Mortalidade. Análise de séries temporais. Epidemiologia. 
Abstract

Objectives: To describe mortality evolution of malnutrition in elderly in Rio de Janeiro (RJMA) and São Paulo Metropolitan Areas (SPMA); to verify it's tendecies, between 19801996; and to propose a model wich permit us to foresee the cases occurances.

Methodology: It's a time series analysis, using ecological data. People from RJMA and SPMA around 60 year-old and older ones, of both sexes were included in this research. The source of those data were the Mortality Information System (1980-1998). The series of monthly cases were analyzed, in a period from January of 1980 to December of 1996. To model the series and to predict the malnutrition cases in elderly. The method Box and Jenkins was used, SARIMA (Seasonal Autoregressive Integrated Moving Average). Results: The results show the existence of seasonable pattern with the largest number of deaths during the months of June and July in SPMA probably because of the cold weather in São Paulo in the winter and during the month of january, in Rio de Janeiro, probably because of the high temperature in this city in the summer. These results can be explained by the difficult of elderly to mainten their bodies in a good temperature during those months what represents an additional stress to their organism poor in nutritional reservations.

Conclusions: The series presented a rising tendency of death and showed seasonable pattern in winter in SPRM and in the summer in RJRM. To estimate the SARIMA models to the Regions for the year of 1996, the results reforced the hypothesis that this method can be efficient to make future forecasts. It's probably that a research wich considers so many death causes will permit to verify the real contribution of the malnutrition as the basic cause or at least a contributive cause of those deaths.

Keywords: Malnutrition. Aged. Mortality. Time series analysis. Epidemiology.

\section{Introdução}

O Brasil entra no terceiro milênio com uma perspectiva de envelhecimento da população que deverá situá-lo entre as nações do mundo com maior número de idosos, e fazê-lo subir da $16^{\text {a }}$ posição, em 1950, para a $6^{\text {a }}$ posição, em 2025, com 31,8 milhões de pessoas com mais de 60 anos $^{1,2}$.

De acordo com o Censo de 1991, as maiores proporções de idosos brasileiros encontram-se em alguns Estados da Região Sudeste (Rio de Janeiro, São Paulo e Minas Gerais) e da Região Nordeste (Paraíba). No Rio de Janeiro e na Paraíba os índices são superiores a 9\%, embora no Rio de Janeiro o índice elevado seja associado ao envelhecimento de sua estrutura etária, e na Paraíba, à perda de indivíduos jovens, à migração ${ }^{3}$.

O distúrbio nutricional mais importante observado nos idosos é a desnutrição proteico-calórica (DPC), que está associada ao aumento da mortalidade, ao aumento da susceptibilidade às infecções e à redução da qualidade de vida. Entretanto, a DPC é freqüentemente ignorada pois é vista, erroneamente, como parte do processo normal de envelhecimento ${ }^{4-7}$.

A determinação do estado nutricional de idosos abrange uma complexa rede de fatores, que inclui questões econômicas, sociais, biológicas, psíquicas e alimentares ${ }^{8,9}$.

A DPC é um problema multinutricional ${ }^{10}$ e pode ser definida como um estado mórbido secundário a uma deficiência, relativa ou absoluta, de um ou mais nutrientes essenciais, que se manifesta clinicamente ou é detectada por meio de testes bioquímicos, antropométricos ou clínicos ${ }^{6,11}$. Ela pode também ser definida por um conjunto de distúrbios clínicos causados por vários graus de deficiência relacionados à tensão e traumas fisiológicos. No marasmo (má nutrição quantitativa, hipoalimentação global ou calórica, subnutrição) há falta de calorias e de proteínas. No Kwashiokor (má nutrição qualitativa, predominantemente protéica) ocorrem distúrbios metabólicos diversos em estados mais graves. Para unificá-los, convencionou-se estabelecer o título gené- 
rico de desnutrição proteico-calórica ${ }^{12}$.

Na última década, estudos populacionais de âmbito nacional ${ }^{2}$, regional ${ }^{13}$ ou municipal $^{14,}$ sobre estado nutricional e perfil antropométrico, permitiram conhecer a situação nutricional dos idosos no país.

Recentemente, um estudo descritivo, realizado na Escola Nacional de Saúde Pública, verificou que no Brasil, entre 1980 e 1997 ocorreram 36.955 óbitos por desnutrição em idosos. Desse total, a maioria ocorreu na faixa etária de 70 anos e mais. A Região Sudeste contribuiu com $64,9 \%$ de todas as mortes, com destaque para as Regiões Metropolitanas dos Estados de São Paulo e Rio de Janeiro, com populações mais numerosas, e maiores valores e proporções, dentre todas as demais localidades da Região Sudeste e do Brasil como um todo ${ }^{15}$.

Esses resultados apontaram para a necessidade de se realizar um segundo artigo específico de análise de séries temporais, que pudesse auxiliar no melhor entendimento do comportamento desses óbitos. Uma vez que ainda existe, em nosso país, uma reduzida experiência/motivação para estudar os idosos/desnutrição por parte dos profissionais de saúde, estudos como esses, tornam-se relevantes. De uma maneira geral, a participação de profissionais de saúde coletiva na discussão das questões do envelhecimento em nosso país é ainda escassa, possivelmente pelo desconhecimento do enorme cam- po de investigação, além do baixo investimento e da falta de prioridade à pesquisa ${ }^{16}$.

Neste artigo, a análise de séries temporais tem como objetivos descrever a evolução da mortalidade por desnutrição em idosos nas Regiões Metropolitanas dos Estados do Rio de Janeiro e São Paulo, verificar as suas tendências, entre 1980 e 1996, e propor um modelo que permita prever a ocorrência de casos.

\section{Metodologia}

Trata-se de um estudo ecológico, temporal, baseado em dados secundários.

O estudo contemplou os registros de óbitos de indivíduos com 60 anos de idade ou mais, de ambos os sexos, das Regiões Metropolitanas dos Estados do Rio de Janeiro e São Paulo.

A fonte de dados foi o Sistema de Informações sobre Mortalidade ${ }^{17}$ (SIM), banco armazenado pelo Ministério da Saúde, através de seus órgãos, Secretaria Executiva/ DATASUS e Fundação Nacional de Saúde/ CENEPI, colocados à disposição em CDROM, para os anos de 1979 a 1996.

Para classificar os óbitos empregou-se a Classificação Internacional de Doenças- CID9, para os anos de 1980 até 1995, e a CID-10 para os anos mais recentes. Desta forma, foram selecionadas as seguintes causas básicas de mortalidade:

\begin{tabular}{lcc}
\hline Causas básicas de mortalidade & $\begin{array}{c}\text { Classificação } \\
\text { Internacional de } \\
\text { Doenças - CID-9 }\end{array}$ & $\begin{array}{c}\text { Classificação } \\
\text { Internacional de } \\
\text { Doenças - CID-10 }\end{array}$ \\
\hline Kwashiokor & 260 & E40 \\
Marasmo Nutricional & 261 & E41 \\
Kwashiokor Marasmático & 262 & E42 \\
Outras formas de DPC grave & & E43 \\
DPC grave não especificada & 263.0 & E44.0 \\
DPC grau moderado & 263.1 & E44.1 \\
DPC grau leve & 263.2 & E45 \\
Interrupção do desenvolvimento & & \\
consequência DPC & 263.8 & E46 \\
Outros tipos de DPC & 263.9 & \\
Tipos não especificados & &
\end{tabular}


Analisou-se a série de casos mensais, no período de janeiro de 1980 a dezembro de 1996, de mortes por desnutrição (CID) em idosos de 60 anos e mais, nas Regiões Metropolitanas de São Paulo e Rio de Janeiro.

Para caracterizar as séries como temporais, ordenaram-se os tempos de observação e criou-se uma variável, o tempo, iniciando com a unidade, e aumentando de uma unidade, seqüencialmente, a cada data de ocorrência. Com o intuito de criar uma série estacionária, isto é, mantendo a variância e a tendência constantes, procedeu-se à transformação log-linear dos casos, com um "lag" de diferença.

Segundo Morettin e Toloi ${ }^{18}$ (1987), existem dois procedimentos usuais para estimar um padrão sazonal, o Método de Regressão e o Método de médias móveis. Desta forma, optou-se por utilizar o primeiro, a partir das médias de casos. A variável dependente correspondeu ao número de casos e a independente ao tempo ordenado; os meses do ano foram considerados como variáveis dummy, tomando como base o mês de dezembro. Optou-se por um nível de significância de 5\%.

Para modelagem da série e predição de casos por desnutrição em idosos, utilizouse o método Box and Jenkins, SARIMA (Sazonal Autoregressive Integrated Moving Average), aplicando-se o software SPSS 9.01998.

Com a estimação dos parâmetros através da função de máxima verossimilhança, realizaram-se correlogramas das funções de autocorrelação (acf) e de autocorrelação parcial (pacf), cujas características forneceram indicações importantes sobre a ordem dos modelos a serem ajustados.

A sazonalidade foi testada através do processo de modelagem. As ordens dos modelos ajustados, através da modelagem SARIMA, forneceram parâmetros que foram comparados entre si. Os parâmetros analisados foram: a significância, o logaritmo da razão de verossimilhança, os critérios de informação Akaike e bayesiano de acordo com Schwartz e os valores estimados para 1996.

A escolha do melhor modelo é uma in- terpretação que o pesquisador faz do conjunto desses parâmetros. Um modelo é considerado bom quando utiliza no processo de modelagem um menor número de parâmetros, com valores de $p$ significativos, e valores dos critérios (Akaike e Bayesiano) e de logaritmo da verossimilhança mínimos possíveis (Wey ${ }^{19}$ 1994). Privilegiou-se neste estudo selecionar os modelos que apresentaram o menor número de parâmetros com significância de $5 \%$ e que resultaram em estimativas de predição mais precisas.

\section{Resultados}

A Figura 1 mostra a série de casos das Regiões Metropolitanas de São Paulo e do Rio de Janeiro, segundo o tempo. No gráfico 1A, pode-se observar na Região Metropolitana de São Paulo a existência de uma grande variância, com registro de aumento até abril de 1988, com queda posterior, seguida de um pico, novamente, em julho de 1996. No gráfico 1B, observa-se na Região Metropolitana do Rio de Janeiro um aumento dos óbitos que culmina num pico no meio do período (1988), ano em que ocorreu o maior número de óbitos desta Região, com tendência de estabilização.

A Tabela 1 mostra o total de casos ocorridos a cada mês, nas duas Regiões Metropolitanas e a média de óbitos em cada um desses meses. Observou-se uma maior concentração de casos nos meses de junho (27) e julho (29) na RMSP e na RMRJ, nos meses de janeiro e agosto (34) e em julho (33).

Utilizando o método de Regressão com as médias de cada mês nas duas Regiões Metropolitanas, observou-se que em São Paulo os meses de junho e julho obtiveram coeficientes com valores de pestatisticamente significativos. No Rio de Janeiro, o mês de janeiro foi aquele que teve coeficiente significativo, ressaltando, também, o mês de agosto com um $p$ valor $(0,066)$ próximo ao ponto de corte considerado $(0,05)$.

O processo de modelagem é iniciado através de transformações, do tipo diferenciação, que transformam a série em estacionária. Segue-se a identificação do modelo, 
$1 A$

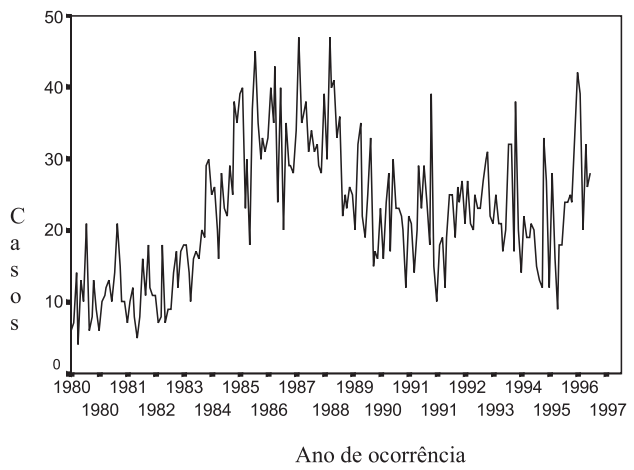

$1 B$

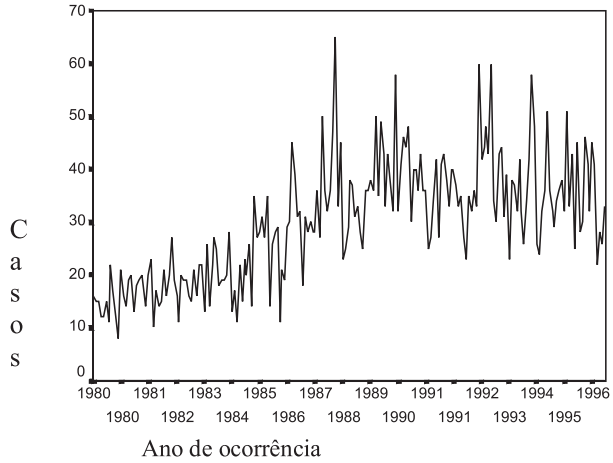

Fonte:DATASUS/ CENEPI -MINISTÉRIO DA SAÚDE, 1998.

Figura 1 - Série de casos mensais, no período de janeiro de 1980 a dezembro de 1996, de óbitos por desnutrição (CID) em idosos, de 60 anos e mais, nas Regiões Metropolitanas de São Paulo (2.1A) e do Rio de Janeiro (2.1B).

Figure 1 - Monthly case series, from january 1980 to december 1996, of deaths due to malnutrition (ICD) in the elderly, 60 years old or more, in the metropolitan areas of São Paulo $(2.1 \mathrm{~A})$ and rio de Janeiro (2.1B).

Tabela 1 - Total de casos e média mensal dos óbitos por desnutrição em idosos na Regiões Metropolitanas de São Paulo e do Rio de Janeiro, por mês, 1980 a 1996.

Table 1 - Total cases and monthly average of deaths due to malnutrition in the elderly, in the metropolitan areas of São Paulo and Rio de Janeiro, 1980 to 1996.

\begin{tabular}{lcccc}
\hline & \multicolumn{2}{c}{ São Paulo } & \multicolumn{2}{c}{ Rio de Janeiro } \\
Meses & Total de Casos & Média Mensal & Total de Casos & Média mensal \\
\hline Janeiro & 357 & 21 & 578 & 34 \\
Fevereiro & 335 & 20 & 475 & 28 \\
Março & 323 & 19 & 489 & 29 \\
Abril & 355 & 21 & 445 & 26 \\
Maio & 410 & 24 & 491 & 29 \\
Junho & 453 & 27 & 504 & 30 \\
Julho & 493 & 29 & 560 & 33 \\
Agosto & 421 & 25 & 581 & 34 \\
Setembro & 384 & 23 & 497 & 29 \\
Outubro & 405 & 24 & 490 & 29 \\
Novembro & 317 & 19 & 487 & 29 \\
Dezembro & 327 & 19 & 500 & 29 \\
\hline Total & 4579 & & 6097 & \\
\hline
\end{tabular}

Fonte/Source: DATASUS/CENEPI-MS, 1998.

com a função de autocorrelação, aplicada ao processo autoregressivo e de médias móveis. A Figura 2 e a Figura 3 mostram o correlograma com as funções de autocorrelação (ACF) e autocorrelação parcial (PACF), com o componente sazonal da série dos óbitos por desnutrição em idosos na Região Metropolitana de São Paulo e do Rio de Janeiro, respectivamente. Verifica-se uma autocorrelação diferente de zero nos lags 1 , 11, 12 e 13 (Figura 2A e Figura 3A). Nas duas séries existe um padrão de quedas expo- 


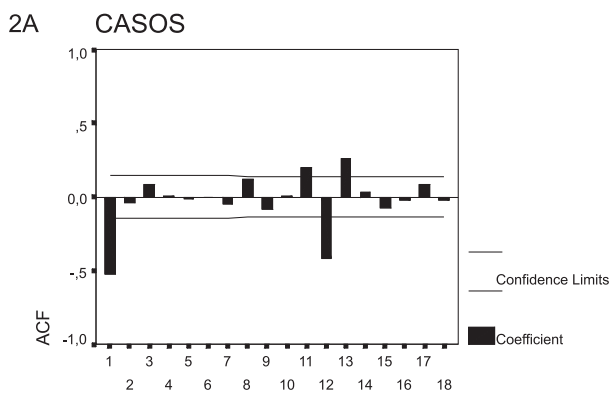

Lag Number

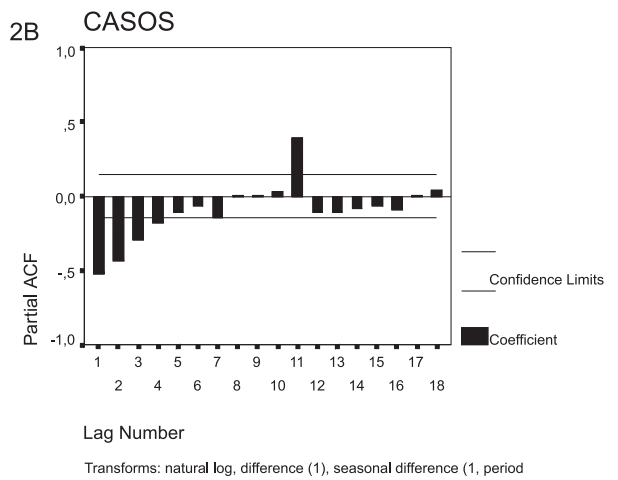

Figura 2 - Autocorrelação e Autocorrelação parcial da série dos óbitos por desnutrição em idosos na Região Metropolitana de São Paulo - 1980-1996.

Figure 2 - Autocorrelation and partial Autocorrelation of the series of deaths due to malnutrition in the elderly in metropolitan São Paulo - 1980-1996.
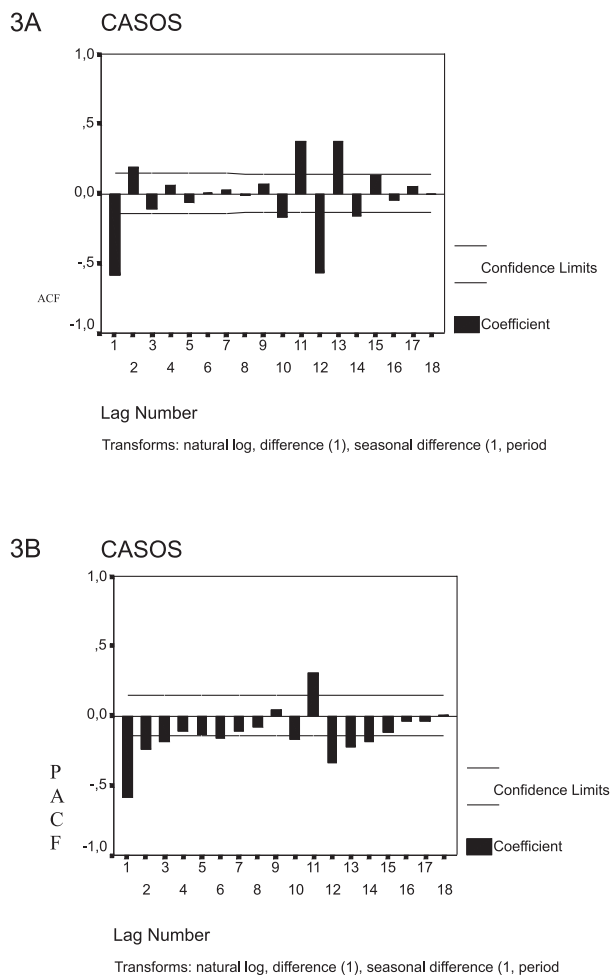

Figura 3 - Autocorrelação e Autocorrelação parcial da série dos óbitos por desnutrição em idosos na Região Metropolitana do Rio de Janeiro - 1980-1996.

Figure 3 - Autocorrelation and partial autocorrelation of the series of deaths due to malnutrition in the elderly in metropolitan Rio de Janeiro - 1980 - 1996.

Tabela 2 - Método de Regressão para estimar o padrão de sazonalidade dos óbitos por desnutrição em idosos nas Regiões Metropolitanas de São Paulo e do Rio de Janeiro, por mês, 1980 a 1996.

Table 2 - Regression method for estimating seasonal pattern of deaths due to malnutrition in the elderly in the metropolitan areas of São Paulo and Rio de Janeiro, 1980 to 1996

\begin{tabular}{lcccc}
\hline Meses & \multicolumn{2}{c}{ São Paulo } & \multicolumn{2}{c}{ Rio de janeiro } \\
& $\begin{array}{c}\text { Coeficiente não } \\
\text { padronizado }\end{array}$ & $p$ valor & $\begin{array}{c}\text { Coeficiente não } \\
\text { padronizado }\end{array}$ & $p$ valor \\
\hline Janeiro & 2,344 & 0,436 & 6,023 & $\mathbf{0 , 0 3 6}$ \\
Fevereiro & 0,997 & 0,740 & $-0,167$ & 0,954 \\
Março & 0,239 & 0,937 & 0,527 & 0,854 \\
Abril & 2,068 & 0,492 & $-2,192$ & 0,444 \\
Maio & 5,251 & 0,082 & 0,383 & 0,893 \\
Junho & 7,728 & $\mathbf{0 , 0 1 1}$ & 1,018 & 0,722 \\
Julho & 10,028 & $\mathbf{0 , 0 0 1}$ & 4,181 & 0,145 \\
Agosto & 5,740 & 0,058 & 5,286 & 0,066 \\
Setembro & 3,511 & 0,244 & 0,215 & 0,940 \\
Outubro & 4,694 & 0,120 & $-0,327$ & 0,909 \\
Novembro & $-0,536$ & 0,859 & $-0,634$ & 0,824 \\
\hline
\end{tabular}

Fonte/Source:DATASUS/ CENEPI-MINISTÉRIO DA SAÚDE, 1998. 
nenciais em PACF (Figura 2 B e Figura 3 B), iniciado com valor negativo, o que sugere um processo de médias móveis, bem como valores significantes que se alternam, começando com negativo, o que sugere um componente autoregressivo.

Com base nas autocorrelações (ACF) e nas autocorrelações parciais (PACF) iniciouse o processo de identificação da ordem do modelo, na tentativa de encontrar aquele que mais se ajustasse. Desta forma, foram testados vários modelos diferentes para as séries temporais dos casos de cada Região Metropolitana, comparando os resultados, dentre outros, a significância dos parâmetros, o logaritmo da razão de verossimilhanças, os critérios de informação Akaike e baysesiano de Schwartz e os valores estimados para 1996.

A Tabela 3 apresenta os parâmetros utilizados e os diversos modelos testados para a Região Metropolitana do Rio de Janeiro. O modelo SARIMA $(0,1,1)(0,1,1)$ apresentou os menores valores de $\mathrm{p}$, sendo todos significativos para os Termos Médias Móveis 1 $(\mathrm{p}<0,0000)$ e Médias Móveis Sazonal 1 $(\mathrm{p}<0,0001)$ e a constante $(\mathrm{p}=0,0127)$ foi mantida por permitir um melhor ajuste. Desta forma, a série de óbitos apresenta um termo médias móveis e um termo médias móveis sazonal. Ao comparar os critérios Akaike e Baysesiano, observa-se que os valores são muito semelhantes em relação aos demais modelos. Em relação ao log da razão de verossimilhança, o modelo escolhido obteve um valor aceitável em relação aos demais. Percebe-se, nos outros modelos, que a inclusão de um Termo Autorregressivo com ou sem componente sazonal altera os resultados, que passam apresentar um valor de $p$ não significativo e, portanto, foram excluídos do modelo final. A adição de mais um termo médias móveis sazonal, como visto no Modelo $(0,1,1)(0,1,2)$, não foi significativo $(\mathrm{p}=0,2926)$ e por isto foi também foi excluído.

A Tabela 4 apresenta os parâmetros utilizados e os diversos modelos testados para a Região Metropolitana de São Paulo. O modelo SARIMA $(2,1,1)(1,1,0)$ apresentou os menores valores de $\mathrm{p}$, sendo todos significa- tivos para os Termos Autoregressivo 1 $(\mathrm{p}<0,0030)$ e Autoregressivo $2(\mathrm{p}<0,0100)$. Embora a constante tenha sido não significativa, optou-se por mantê-la, pois o modelo revelou melhores valores preditivos. A série apresentou ainda um Termo Médias Móveis $(\mathrm{p}<0,0001)$ e um termo Auto-regressivo Sazonal $(\mathrm{p}<0,0001)$. Desta forma, a série de óbitos da Região Metropolitana de São Paulo difere da Região Metropolitana do Rio de Janeiro por conter 4 parâmetros: 2 termos Autorregressivos, 1 Termo Médias Móveis e 1 Termo Autoregressivo sazonal. Ao comparar os critérios Akaike e Baysesiano dos modelos $(2,1,1)(1,1,0)$, com e sem a constante, observam-se valores maiores para o modelo escolhido, porém a escolha baseouse no fato de o mesmo apresentar melhores valores preditivos. Percebe-se, a partir dos outros modelos, que a inclusão de um termo Autorregressivo com componente sazonal melhora o resultado dos critérios e do log da razão de verossimilhança. Os valores de $p$ foram todos significativos ( $\mathrm{p}<0,05$ ).

Com os modelos escolhidos para cada Região Metropolitana, foram realizadas predições em relação aos óbitos ocorridos no ano de 1996, tomando como base os óbitos ocorridos até o ano anterior, 1995. Foram colocados juntos, na Tabela 5, os valores originais da série e os preditos, para permitir a comparação entre eles e observar se os valores preditos se assemelham aos valores observados (reais), e desta forma, atestar o uso desta técnica neste tipo de estudos. Os valores indicaram uma predição exata do total de casos, para a RMRJ, e valores aproximados, para a RMSP. Quando se consideram os valores mensais, os valores são próximos para ambas as Regiões.

Observa-se que os maiores valores se mantêm na previsão para os meses de junho e julho na RMSP, e para os meses de janeiro e agosto na RMRJ.

A Figura 4a com a série dos casos da RMRJ e a Figura $4 \mathrm{~b}$ com a série dos casos da RMSP mostram as estimativas dos casos com respectivos intervalos de confiança, evidenciando os bons ajustes do modelos. 


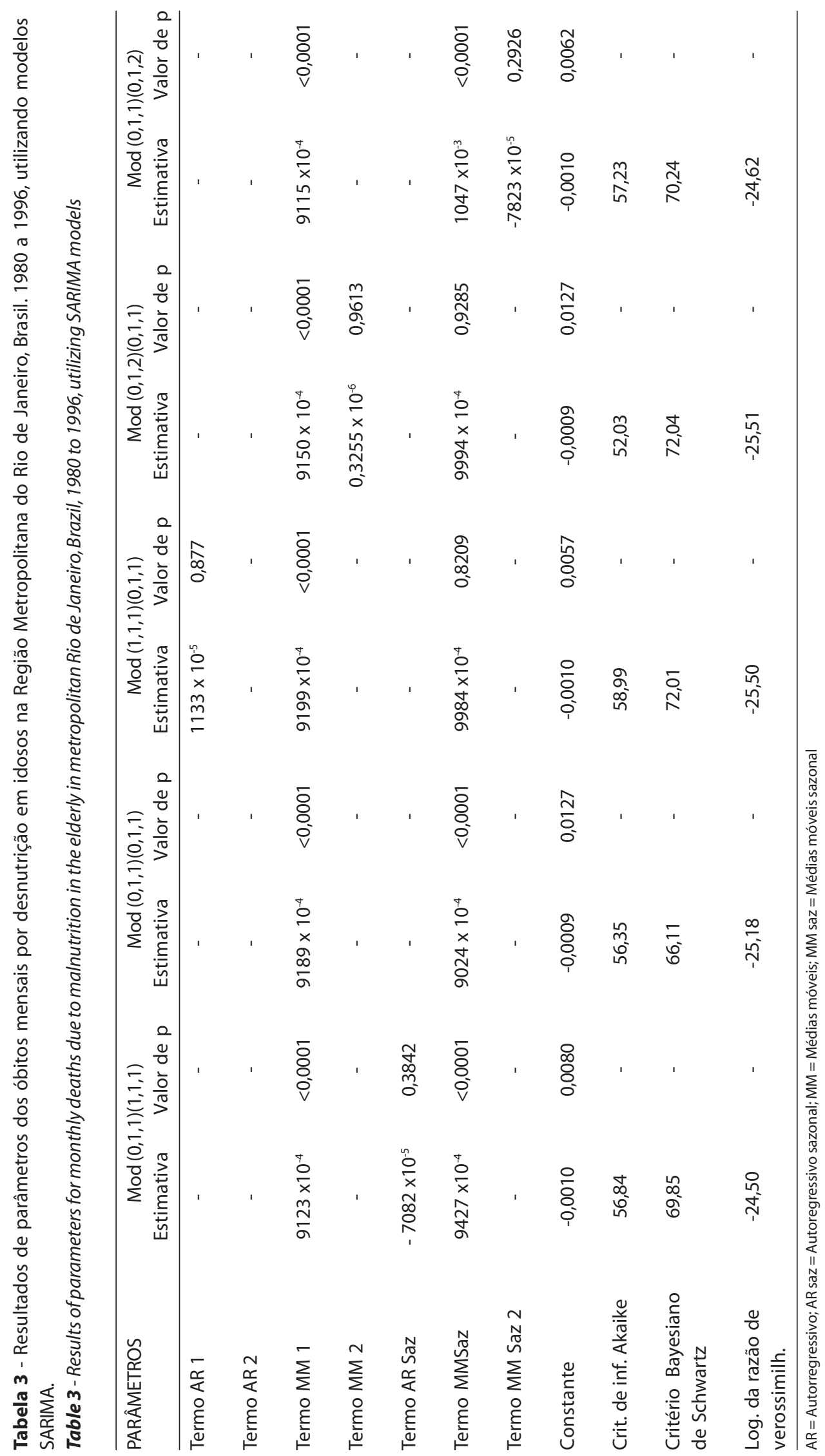




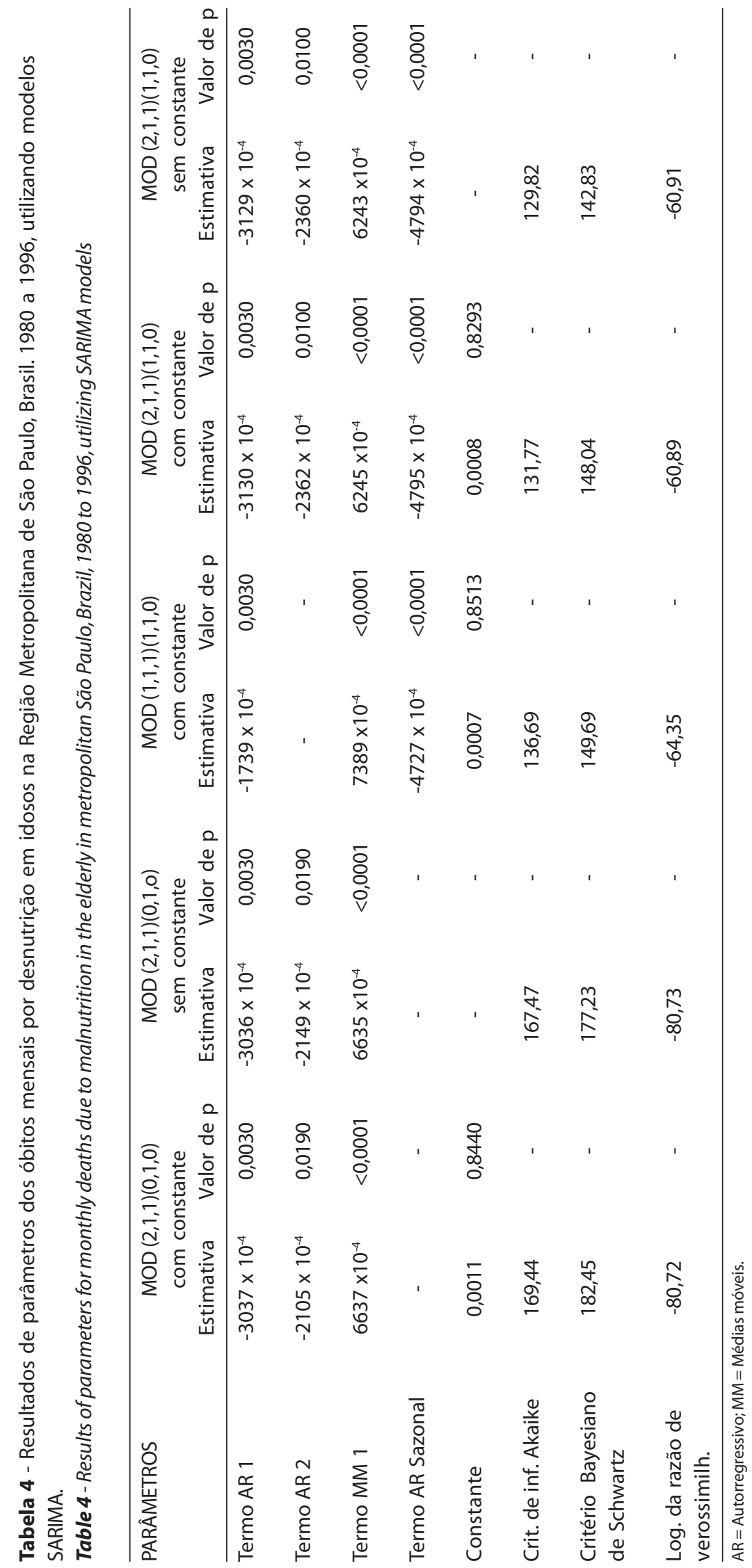


Tabela 5 - Comparação dos valores de óbitos originais por desnutrição e os estimados pelo modelo SARIMA $(2,1,1)(1,1,0)$ na Região Metropolitana de São Paulo e pelo modelo SARIMA $(0,1,1)(0,1,1)$ na Região Metropolitana do Rio de Janeiro. Brasil - 1996

Tabela 5 - Comparison of deaths due to malnutrition and those estimated by the SARIMA model $(2,1,1)(0,1,1)$ in Metropolitan São Paulo and by the SARIMA model $(0,1,1)(0,1,1)$ in Metropolitan Rio de Janeiro. Brazil - 1996

\begin{tabular}{|c|c|c|c|c|c|c|}
\hline \multirow[b]{2}{*}{ Ano de 1996} & \multicolumn{3}{|c|}{ Região Metropolitana de São Paulo } & \multicolumn{3}{|c|}{ Região Metropolitana do Rio de Janeiro } \\
\hline & $\begin{array}{l}\text { Valores } \\
\text { originais } \\
\text { da série }\end{array}$ & $\begin{array}{c}\text { Preditos } \\
\text { por Sarima } \\
(2,1,1)(1,1,0)\end{array}$ & IC( $-95 \%)$ & $\begin{array}{c}\text { Valores } \\
\text { originais } \\
\text { da série }\end{array}$ & $\begin{array}{c}\text { Preditos } \\
\text { por Sarima } \\
(0,1,1)(0,1,1)\end{array}$ & IC( $-95 \%)$ \\
\hline Janeiro & 18 & 17 & $9-32$ & 45 & 40 & $23-67$ \\
\hline Fevereiro & 24 & 13 & $7-25$ & 28 & 35 & $21-58$ \\
\hline Março & 24 & 13 & $7-26$ & 30 & 33 & $20-56$ \\
\hline Abril & 25 & 17 & $9-33$ & 46 & 29 & $17-49$ \\
\hline Maio & 24 & 19 & $10-38$ & 41 & 33 & $20-56$ \\
\hline Junho & 33 & 27 & $14-52$ & 32 & 36 & $22-61$ \\
\hline Julho & 42 & 38 & $19-73$ & 45 & 39 & $23-65$ \\
\hline Agosto & 39 & 19 & $10-36$ & 41 & 40 & $24-67$ \\
\hline Setembro & 20 & 27 & $14-51$ & 22 & 36 & $21-60$ \\
\hline Outubro & 32 & 26 & $13-49$ & 28 & 32 & $19-53$ \\
\hline Novembro & 26 & 20 & $10-39$ & 26 & 32 & $19-54$ \\
\hline Dezembro & 28 & 27 & $14-52$ & 33 & 32 & $19-54$ \\
\hline Total & 335 & 262 & $135-507$ & 417 & 417 & $247-701$ \\
\hline
\end{tabular}

Fonte/Source:DATASUS/ CENEPI -MINISTÉRIO DA SAÚDE, 1998.
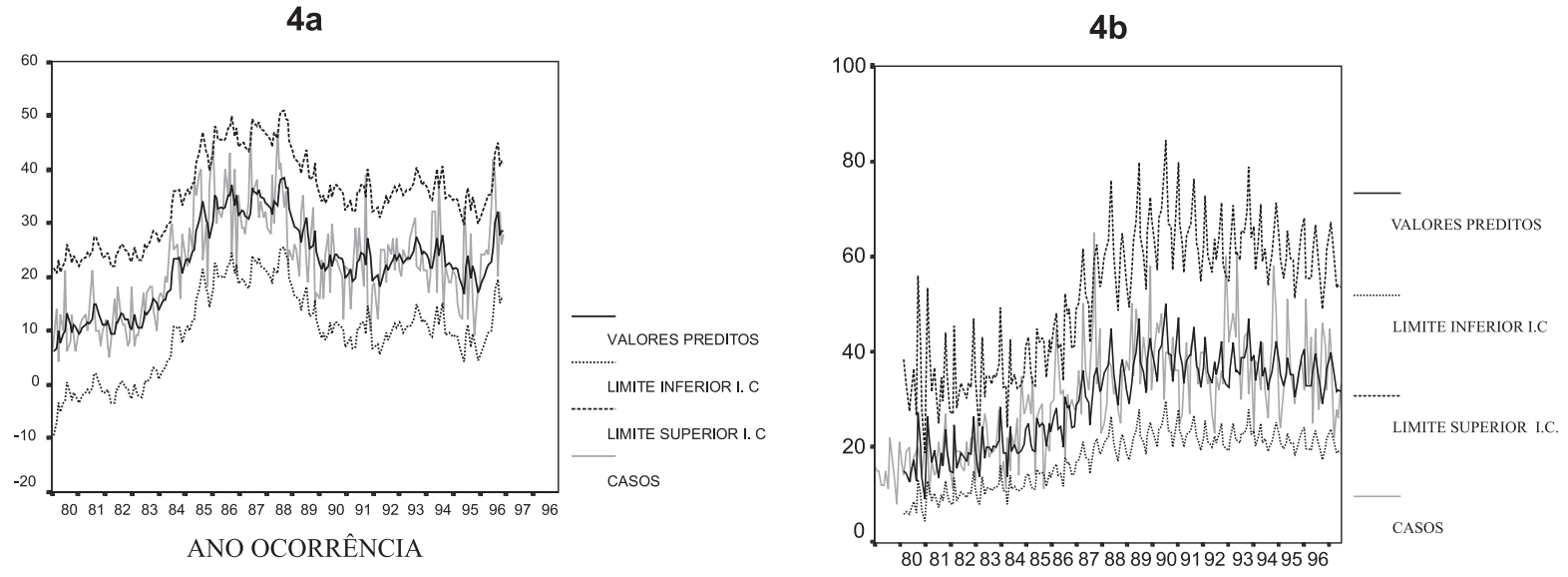

ANO OCORRÊNCIA

Figura 4 - Gráfico das séries originais dos óbitos por desnutrição em idosos nas Regiões Metropolitanas de São Paulo (2.3 a) e |Rio de Janeiro (2.3 b), por mês de 1980 a 1996 e valores preditos pelo modelo SARIMA $(2,1,1)(1,1,0)$ e SARIMA $(0,1,1)(0,1,1)$, respectivamente, com intervalos de confiança .

Figure 4 - Original series of deaths due to malnutrition in the elderly in the Metropolitan areas of São Paulo (2.3 a) and Rio de Janeiro $(2.3$ b), by month, from 1980 to 1996 and values estimanted by the SARIMA model $(2,1,1)(1,1,0)$ and SARIMA $(0,1,1)(0,1,1)$, respectively, and confidence intervals. 


\section{Discussão}

Nos últimos anos, o grupo de idosos tem chamado atenção de diversos atores sociais como políticos, gestores de saúde e epidemiologistas, por ter experimentado um crescimento maior em relação aos demais grupos etários.

O desafio consiste em conhecer melhor essa população e suas características, e dar subsídios para o planejamento de ações e políticas públicas, que invista neste envelhecimento com qualidade de vida. Esta população se caracteriza pela busca regular dos serviços de saúde, que deverão estar preparados para uma demanda cada vez crescente devido às susceptibilidades deste grupo às doenças.

Ao longo dos anos, a desnutrição tem merecido destaque como causa relevante de doença e morte, manifestada principalmente em crianças de países em desenvolvimento. Trata-se de uma situação mórbida, capaz de refletir as condições de vida da população em geral. Programas têm sido implementados com eficiência (Programa do incentivo ao aleitamento materno, da cesta básica, da merenda escolar), na tentativa de prevenir e corrigir este problema. No entanto, o papel da desnutrição como causa de morte na população idosa, bastante discutido internacionalmente, tem sido pouco considerado em estudos brasileiros. Desta forma, este trabalho enfoca a desnutrição como um evento importante na velhice, capaz de levar o paciente idoso ao óbito.

Pacientes idosos, portadores de desnutrição proteico-calórica, apresentam risco aumentado de adquirir doenças e/ou evoluir de forma desfavorável a um tratamento específico $^{20}$. A magreza excessiva da população idosa é apontada como um fator mais fortemente associado à mortalidade do que o excesso de peso.

A escolha das Regiões Metropolitanas de São Paulo e Rio de Janeiro deve-se ao fato de serem as duas localidades da Região Sudeste com o maior contingente de idosos do país. Numa análise preliminar, Otero e colaboradores $^{15}$ (2002), considerando as Regiões
Metropolitanas e Outras Regiões do Sudeste, observaram valores mais elevados de óbitos por desnutrição em idosos nos Estados do Rio de Janeiro e de São Paulo, e valores menores em Minas Gerais e no Espírito Santo, para as proporções. O resultado sugere que as mortes por desnutrição em idosos são fenômenos que atingem os grandes conglomerados urbanos.

Em relação à cobertura dos registros dos óbitos para as Unidades da Federação Brasileira, em 1990 foi classificada como "boa" ou "satisfatória" para todos os Estados do Sul, Sudeste, Centro-Oeste e para os do Nordeste, abaixo do Rio Grande do Norte ${ }^{21}$.

Constata-se uma melhoria na qualidade do sistema dos registros civis, o que permite avaliar com mais segurança os indicadores deles derivados e, conseqüentemente, planejar melhor as ações voltadas para a saúde pública. Esta evidência sugere que não há motivos para negligenciar as estatísticas de óbitos no Brasil. A desculpa de que eles são de má qualidade não serve como pretexto para ignorá-los. Para muitas regiões do Brasil, eles permitem a construção de indicadores de mortalidade válidos ${ }^{21}$.

Segundo os resultados obtidos, a distribuição da série de casos nas duas Regiões Metropolitanas evidenciou a presença de grande variância em ambas as séries, e padrões distintos de ocorrência de casos entre as duas Regiões, com maior número total $\mathrm{e}$ maior média de casos, por mês de ocorrência, na Região Metropolitana do Rio de Janeiro.

Os resultados apontam a existência de sazonalidade, com maior concentração de óbitos nos meses de junho e julho, na Região Metropolitana de São Paulo, e no mês de janeiro, no Rio de Janeiro, provavelmente decorrente do clima frio, em São Paulo, e do calor intenso no Rio de Janeiro. Os valores de significância limítrofes do mês de agosto no Rio de Janeiro, resultantes talvez do tamanho amostral, levantam a suspeição de que também o clima frio deste mês possa contribuir para o maior número de casos. Este resultado pode ser explicado pela possibilidade de idosos, com estado nutricional comprometi- 
do, terem dificuldades em manter a temperatura corporal normal durante os meses de inverno, representando um estresse adicional a um organismo sem reservas. Segundo Tierney ${ }^{11}$, em 1996, a mortalidade no frio é elevada entre a população idosa e tem possivelmente um elo com a desnutrição. O baixo peso danifica a proteção térmica corporal, aumenta o risco de baixa temperatura no inverno e, possivelmente, contribui para as quedas e as doenças.

A relação entre desnutrição e doença respiratória é reconhecida há muito tempo. Durante períodos de fome, as infecções respiratórias são complicações freqüentes da inanição $0^{22}$.

A estrutura e função do parênquima pulmonar são alterados pela desnutrição. O tecido pulmonar pode ser mais suscetível à lesão e o processo de reparação é interrompido nos estados de desnutrição. A complacência aumentada e a elasticidade diminuída no pulmão são produzidas pela desnutrição, podendo prejudicar a função pulmonar. A hipoproteinemia resultante da desnutrição contribui para o desenvolvimento de edema pulmonar ${ }^{22}$.

A associação da desnutrição com rebaixamento da imunidade coloca o paciente desnutrido com doença pulmonar em risco para desenvolvimento de infecções respiratórias ${ }^{22}$.

Por outro lado, no verão o organismo necessita maior quantidade de água, para evitar a desidratação, que pode ocorrer em idosos desnutridos. A água é essencial aos processos fisiológicos da digestão, absorção e excreção de resíduos metabólicos, e ao funcionamento do sistema circulatório. A desnutrição proteico-calórica, a doença, o traumatismo ou a cirurgia podem causar alteração na quantidade e na composição de fluidos teciduais, e resultar em desidratação, choque e morte $^{10}$.

Num estudo sobre a influência dos fatores ambientais nos atendimentos de emergência, utilizando modelagem ARIMA, houve associação entre mudanças na temperatura e aumento do número de registros hospitalares por causas respiratórias, no inver- no, especialmente para aqueles com idade acima de 65 anos. Também foi registrado o aumento de casos no verão, relacionado ao efeito do calor, principalmente sobre as enfermidades do aparelho circulatório ${ }^{23}$.

Um outro estudo sobre mortalidade e sua relação com a temperatura do ar, realizado pelo Centro Universitário de Saúde Pública de Madri, utilizando modelagem ARIMA para variáveis de temperatura e de mortalidade, mostrou que existe uma associação estatísticamente significativa entre a mortalidade e os extremos térmicos. $\mathrm{O}$ estudo acrescenta que a resposta fisiológica às mudanças de temperatura, especificamente as mais altas, no grupo de 45-64 anos, é melhor do que para os idosos com mais de 65 $\operatorname{anos}^{24}$.

A existência de um valor positivo significativo para a mortalidade total e a temperatura indica que a elevação da temperatura vem associada a um incremento da mortalidade e, por outro, que os efeitos do calor sobre a mortalidade são imediatos, o que é coerente com os mecanismos biológicos que relacionam as altas temperaturas com um incremento da viscosidade do sangue e do colesterol sérico ${ }^{24}$.

A técnica de análise de séries temporais é uma ferramenta útil, porém com sistemáticas para modelagem e interpretações de resultados nem sempre triviais. Embora a distribuição dos casos e a análise dos correlogramas forneçam pistas introdutórias para os modelos a serem constituídos, as múltiplas combinações de parâmetros com diversos resultados de significância dos estimadores, a proximidade dos valores de deviância, critério de AKAIKE etc, colocam o pesquisador em um dilema de escolha do melhor modelo. Certamente esta escolha está relacionada com o objetivo do trabalho. Como neste caso, pretendia-se estimar casos para anos subsequentes, a predição foi fator relevante para tal escolha.

Ao estimar os modelos SARIMA para as Regiões Metropolitanas de São Paulo e Rio de Janeiro para o ano de 1996, podem-se comparar os dados originais da série com os valores preditos. Observam-se valores pró- 
ximos aos originais, embora alguns tenham um intervalo de confiança amplo. Este achado reforça a hipótese de que este método pode ser eficiente para fazer previsões futuras, permitindo, assim, melhor planejamento e intervenção em Saúde, Pública. Em particular, saber qual o número esperado de óbitos por desnutrição nos próximos anos possibilita monitorar e planejar estratégias de intervenção, uma vez que, segundo a $\mathrm{OMS}^{25}$ (1995), no contexto de risco de desnutrição aguda, os idosos podem servir de sentinela para toda a população. Segundo Helfenstein ${ }^{26}$ (1996), as previsões de séries temporais são importantes, pois permitem aos serviços de saúde pública conhecer a freqüência esperada das doenças e, assim, melhorar o planejamento na distribuição dos recursos. Esta técnica pode também ser utilizada como complementar ao método de análise de intervenção. Uma previsão obtida dos dados antes da intervenção pode ser comparada com a atual obtida depois da intervenção ${ }^{26}$.

Este estudo traz uma temática ainda não abordada de forma específica em outros trabalhos da área, porém, apresenta algumas limitações que devem ser consideradas ao discutir os resultados encontrados.

Cabe destacar a limitação do espaço geográfico, uma vez que foram incluídas apenas duas Regiões Metropolitanas da Região Sudeste, o que não permite extrapolação para o restante do país, dadas as suas diferenças demográficas, culturais, políticas e sócioeconômicas.

Sabe-se que a subnotificação de registros no país é relevante, mesmo no SIM onde a cobertura é considerada boa. Desta forma, é possível que haja mais casos de desnutrição em idosos (não declarados) ou menos casos (erro). Ainda que seja "surpreendente” a ocorrência de causa básica por desnutrição em idosos, certamente um estudo que levasse em consideração causas múltiplas de morte permitiria verificar a real contribuição da desnutrição como causa básica ou contributiva para a morte. Infelizmente, até este momento, esses dados não estão disponíveis para análise.
Pode-se considerar, ainda, o reduzido número de casos mensais registrado em alguns meses. Segundo Helfenstein ${ }^{26}$ (1996), isso constitui um problema quando se trabalha com análise de séries temporais.

\section{Conclusões}

O Brasil tem experimentado uma mudança no perfil de saúde da população, com predomínio das doenças crônicas, fato observado mais acentuadamente nos últimos anos. A desnutrição no grupo de idosos começa a despertar o interesse, pelo número expressivo de óbitos observados a cada ano, principalmente entre os mais idosos. Alguns estudos revelam que a desnutrição surge como fator independente de morbidade, ou seja, independente de uma patologia crônica como câncer, insuficiência cardíaca, acidente vascular cerebral etc. Desta forma, hipóteses sobre a etiologia da doença podem ser levantadas, pois a desnutrição em idosos tanto pode ser resultado de carências nutricionais crônicas, como pode ter relação com um quadro carencial agudo.

Este trabalho considerou duas grandes metrópoles brasileiras, com características semelhantes em relação a fatores socioeconômicos e de acesso a serviços. Num estudo anterior, verificou-se um maior número de óbitos nestas duas localidades em relação às demais da Região Sudeste e do Brasil como um todo. Considerando que essas Regiões dispõem de tecnologias mais modernas e um maior número de unidades de saúde para o atendimento da população, como explicar esses óbitos?

Uma possível explicação é o despreparo dos serviços de saúde no atendimento à demanda e certa ineficácia no serviço prestado ao idoso. Questiona-se a importância atribuída à desnutrição, isto é, se há peocupação dos serviços em realizar uma avaliação nutricional dos idosos, hospitalizados ou não. Sabe-se que, em alguns países, os idosos são acompanhados através de estudos de coorte e de surveys periódicos, que conseguem detectar o problema da desnutrição no idoso e intervir. Parece que nos falta, en- 
tão, um olhar mais direcionado a esta população, buscando alternativas de monitoramento do seu estado de saúde. É preciso que eventos preveníveis e de fácil resolução, como a desnutrição leve, sejam combatidos por medidas simples e de baixo custo, e evitar que evoluam e tomem proporções maiores, agravando o estado de saúde do idoso e predispondo-os a outras enfermidades e ao óbito.

Vendo por outro prisma, independente da etiologia, o problema dos óbitos por desnutrição em idosos incorre em outras questões importantes sob a ótica social, que não culpabilizam diretamente os serviços: a desigualdade socioeconômica e o papel das políticas públicas.

Para compreender a posição dos idosos pobres na distribuição de renda, Barros e Santos $^{27}$ (1999) analisaram os dados da PNAD de 1977; esses autores concluíram que a renda domiciliar cresce com a idade até os 60 anos, para depois declinar, e que as rendas de aposentadoria constituem a parcela principal da renda domiciliar, sendo $60 \%$ da mesma entre os idosos pobres. Também sugerem a existência de maior desigualdade de renda entre os idosos do que entre os não idosos. Eles demonstram que a presença do idoso numa família reduz o grau de pobreza da mesma e que a pobreza entre os não idosos seria maior, caso os idosos constituíssem domicílios separados.

Considerando, então, que a renda dos idosos contribui para diminuir o grau de pobreza dos mais pobres, é possível pensar no impacto dessa contribuição na qualidade da alimentação do idoso que, dadas as suas peculiares condições de saúde, teria uma demanda maior de elementos protéicos na dieta, e a necessidade de garantir uma alimentação mais saudável, incluindo dietas especiais, sabidamente mais onerosas.

Outro aspecto importante, relacionado à questão socioeconômica, é que os idosos brasileiros residentes com seus familiares pertencem a domicílios cuja renda total não ultrapassa três salários-mínimos. A falta de recursos se agrava, uma vez que não existem políticas sociais abrangentes de suporte aos cuidadores em setores como a alimentação, auxílio domiciliar, assistência médica e outros serviços. Ressalta-se, também, que à medida em que a população envelhece aumenta a demanda por instituições de longa permanência ${ }^{28}$.

Em relação ao padrão sazonal dos óbitos por desnutrição, observado neste estudo, é revelador que para este agravo de saúde a atenção deva ser redobrada nos idosos no verão, no Rio de Janeiro, e no inverno em São Paulo. Os extremos de temperatura constituem um estresse adicional para o idoso desnutrido.

É preciso, portanto, aprofundar o estudo do papel da desnutrição na população acima dos 60 anos. A presente investigação, de caráter preliminar, levanta algumas questões que caberá esclarecer no futuro. Há tendência de aumento dos óbitos por desnutrição na velhice, na medida em que cresce a esperança de vida, e mantêm-se as desigualdades e o baixo impacto das políticas públicas atuais? Qual o papel da desnutrição como causa associada de mortalidade? Qual o impacto dos custos dos alimentos na mortalidade por desnutrição em idosos, ao longo do tempo?

Observou-se, neste estudo, que análises estatísticas do tipo séries temporais constituem importante ferramenta para o planejamento de ações em saúde, pois permitem fazer previsões de eventos futuros, a partir de dados passados. Os autores concluem pela necessidade de estudos complementares que incluam outras covariáveis, como as socioeconômicas, que auxiliem na melhor compreensão dos fenômenos apontados. 
1. Baeta AMC. Transição demográfica e novas demandas em saúde: o atendimento à terceira idade. $\boldsymbol{R e v} \mathbf{A d m}$ Pública 1991; 25: 173-8.

2. Tavares EL, Anjos LA. Perfil antropométrico da população idosa brasileira. Resultados da Pesquisa Nacional Sobre Saúde e Nutrição. Cad Saúde Pública 1999; 15:759-68.

3. Telarolli RJ, Machado JCMS, Carvalho F. Perfil demográfico e condições sanitárias dos idosos em área urbana do Sudeste do Brasil. Rev Saúde Pública 1996; 30: 485-98.

4. Sullivan DH, Sun S, Walls RC. Protein-energy undernutrition among elderly hospitalized patients. JAMA 1999; 281:2013-9.

5. Wahlqvist ML, Savige SG, Lukito W. Nutritional disorders in the elderly. Med J Aust 1995; 163: 376-81.

6. Guigoz Y, Vellas B, Garry PJ. Assessing the nutritional status of the elderly: The Mini Nutritional Assessment as part of the geriatric evaluation. Nutr $\operatorname{Rev} 1996$; 54 (1 Pt 2) S59- S65.

7. Wilson MG, Vaswani S, Liu D, Morley JE, Miller DK. Prevalence and causes of undernutrition in medical outpatients. Am J Med 1998; 104: 56-63.

8. Rolandelli RH, Ullrich JR. Suporte Nutricional no Paciente Cirúrgico Idoso debilitado. Clin Cir Am Norte. 1994; 1.

9. Podrabsky M. Nutrição e envelhecimento. In: Mahan LK, Arlin MT, editores. Krause: alimentos nutrição e dietoterapia. 8a ed. São Paulo: Roca; 1995. p. 255.

10. Czaka-Narins DM. Avaliação do estado nutricional. In: Mahan LK, Arlin MT, editores. Krause: alimentos, nutrição e dietoterapia. 8a ed. São Paulo: Roca; 1995. p. 309.

11. Tierney JA. Undernutrition and elderly hospital patients: a review. J Adv Nurs 1996; 23: 228-36.

12. Alves DC. Desnutrição. In: Augusto ALP, Alves DC, Mannarino IC, Gerude M., organizadores. Terapia nutricional. São Paulo: Atheneu; 1995. p.220.

13. Martins SM, Meléndez GV, Cervato AM. Estado nutricional de grupamentos sociais da área metropolitana de São Paulo, Brasil. Cad Saúde Pública 1999; 15: 71-8.

14. Pereira RA. Avaliação antropométrica do estado nutricional. In: Rosely Sichieri, organizadora.

Epidemiologia da obesidade. Rio de Janeiro: UERJ; 1998.

15. Otero UB, Rozenfeld SL, Gadelha AJ, Carvalho MS. Prevalência de óbitos por desnutrição em idosos, Região Sudeste, 1980-1997. Rev Saúde Pública 2002; 36:141-8.
16. Veras RP, Alves, MIC. A população idosa no Brasil: considerações acerca do uso de indicadores de saúde. In: Minayo MC. Os muito brasis: saúde e população na década de 80. Rio de Janeiro: Abrasco; 1995. p. 320 37.

17. Ministério da Saúde. Sistema de Informação Sobre Mortalidade [CD-ROM]. Brasília (DF): MS/DATASUS/ Fundação Nacional de Saúde/CENEPI; 1999.

18. Morettin PA, Toloi CM. Previsão de séries temporais. 2.ed. São Paulo: Atual Editora; 1987. p. 64.

19. Wey WWS. Time series Analysis: univariate and multivariate methods. Reading: Addison-Wesley; 1994.

20. Marchini JS, Ferriolli E, Moriguti JC. Suporte nutricional no paciente idoso: definição, diagnóstico, avaliação e intervenção. Medicina (Ribeirão Preto) 1998; 31: 5461.

21. Paes NA, Albuquerque MEE. Avaliação da qualidade dos dados populacionais e cobertura dos registros de óbitos para as regiões brasileiras. Rev Saúde Pública 1999; 33: 33-43.

22. Adams E. Cuidado nutricional na doença pulmonar. In: Mahan LK, Arlin MT, editores. Krause: alimentos, nutrição e dietoterapia. 8a ed. São Paulo: Roca; 1995. p. 616 .

23. Ferrón MBL, Jiménez JD, Otero JJG, Ortiz, MSP, Odriozola, JCA. Influencia de los factores ambientales en el número de ingresos por urgencias en el complejo hospitalario "Juan Canalejo" de a Coruña: elaboración de un modelo de predicción. Rev Esp Salud Pública 1999; 73:1.

24. Pajares Ortiz MS, Diaz Giménez J, Montero Rubio JC, Alberdi Odriozola JC, Mirón Pérez IJ. Mortalidad diaria em la comunidad de Madrid (1986-1991) para el grupo de 45 a 64 años: su relación com la temperatura del aire. Rev Esp Salud Pública 1997; 71: 149-60.

25. World Health Organization. Physical status: the use and interpretation of anthropometry. Geneva: WHO; 1995. p. 375-411. (WHO-Technical Report Series, 854).

26. Helfenstein U. Box-Jenkis modelling in medical research. Stat Methods Med Res 1996; 5:3-22.

27. Barros RP, Santos RMD. Incidência e natureza da pobreza entre idosos no Brasil. In: Camarano AA, organizadora. Muito além dos 60: os novos idosos brasileiros. Rio de Janeiro: IPEA; 1999. p. 221-50.

28. Chaimowicz FA. Saúde dos idosos brasileiros às vésperas do século XXI: problemas, projeções e alternativas. Rev Saúde Pública 1997; 31: 184-200. 